\title{
Direct Large-Scale $N$-Body Simulations of Planetesimal Dynamics
}

\author{
Derek C. Richardson, Thomas Quinn, Joachim Stadel, and George Lake \\ Department of Astronomy, University of Washington, Box 351580, Seattle, Washington 98195-1580 \\ E-mail: dcr@astro.washington.edu
}

Received September 14, 1998; revised May 3, 1999

\begin{abstract}
We describe a new direct numerical method for simulating planetesimal dynamics in which $N \sim 10^{6}$ or more bodies can be evolved simultaneously in three spatial dimensions over hundreds of dynamical times. This represents several orders of magnitude improvement in resolution over previous studies. Theadvance is made possible through modification of a stable and tested cosmological code optimized for massively parallel computers. However, owing to the excellent scalability and portability of the code, modest clusters of workstations can treat problems with $N \sim 10^{5}$ particles in a practical fashion.

The code features algorithms for detection and resolution of collisions and takes into account the strong central force field and flattened K eplerian disk geometry of planetesimal systems. Wedemonstrate the range of problems that can be addressed by presenting simulations that illustrate oligarchic growth of protoplanets, planet formation in the presence of giant planet perturbations, the formation of the jovian moons, and orbital migration via planetesimal scattering. We also describe methods under development for increasing the timescale of the simulations by several orders of magnitude. (c) 2000 Academic Press
\end{abstract}

Key Words: $N$-body simulation; planetesimals; planetary formation; planetary dynamics; resonances.

\section{INTRODUCTION}

We begin with an overview of the current issues in planet formation, emphasizing how direct simulations of planetesimal dynamics can help address these issues. This is followed by a summary of previous work by other researchers, focusing on direct simulation methods and their current limitations. Section 2 explains in detail our new approach to the challenge of direct simulation, while Section 3 presents several examples illustrating the new technique. We conclude with a discussion of future improvements to the code and related applications in Section 4.

\subsection{Outstanding Issues in Planet Formation}

Studies of planet formation are faced with a variety of fundamental questions:

What are the planet formation timescales? The timescales are sensitive to the initial mass distribution and the nature of the growth processes (i.e., whether there was a period of run- away growth-see below). However, there are important observational constraints. For example, pre-main-sequence stars lose their infrared excesses in $\sim 1-10 \mathrm{Myr}$ (Strom et al. 1993), setting a limit to the timescale for the planetesimals to become large enough to cease "grinding" collisions that return dust to the disk. Subsequent evolution from these protoplanets to planets in the inner Solar System may take significantly longer $\left(\sim 10^{8} \mathrm{yr}\right.$; Chambers and Wetherill 1996). The transition from rapid growth to long-term interactions has been treated only qualitatively so far (Lissauer 1993). One of our major goals is to observe this transition directly with our simulation code.

What was the primordial surface density distribution? By smearing out the known mass in the Solar System and allowing for depletion of volatiles, we can use the current state to guess the initial surface mass density distribution $\Sigma(a)$ in the inner nebula. This is modeled as $\Sigma(a) \propto a^{-\alpha}$, where $a$ is the distance from the Sun, $\alpha$ is in the range of 1 to 2 , and $\Sigma(1 \mathrm{AU}) \sim 10 \mathrm{~g}$ $\mathrm{cm}^{-2}$ (Weidenschilling 1977, Hayashi 1981, Tremaine 1990, Chambers and Wetherill 1998). However, this straightforward approach leads to a significant dip in the vicinity of Mars andas always - the asteroid belt calls out for a special explanation. Ironically, if we attribute the absence of a terrestrial planet in the asteroid belt to gravitational perturbations by Jupiter, then a proto-Jupiter must form very quickly $\left(\lesssim 10^{5} \mathrm{yr}\right.$ ) in order to prevent runaway accretion in the asteroid belt. This may require an excess of mass compared to the "minimum mass" model in the outer Solar System (Lissauer and Stewart 1993). The enhanced disk mass may save conventional giant planet core accretion models that otherwise suffer from formation times in excess of the disk-clearing timescale (Boss 1997). Direct simulations are now close to the point where they can put tighter constraints on the initial mass distribution and the "belt mechanism" by treating $\alpha$ and $\Sigma$ as free parameters and by exploring the final states of models with and without an early Jupiter.

What controls "runaway" growth? The search for runaway growth has been a consistent focus of planetesimal work (Section 1.2). While it is now generally accepted that a few bodies do detach from the general planetesimal mass distribution with accelerated growth rates after a certain amount of time and under certain conditions (e.g., the form of the mass and velocity distribution is important), some of the details remain uncertain. 
This is because direct simulations have to date been too coarse to do more than scratch the surface of the problem. Our ultimate goal with the new code is to have sufficient dynamic range and time coverage to quantify directly the conditions under which runaway growth both begins and ceases to become effective. We report here on our first steps toward that objective. We also wish to examine the extent to which these regimes overlap depending on such factors as orbital distance and external perturbations from other developing embryos or even giant planets.

Was there strong radial mixing? Wetherill's (1990) simulations suggest radial mixing during protoplanet accumulation sufficient to blur chemical gradients-at odds with the dependence of asteroid spectral type on semimajor axis seen by Gradie et al. (1989). Direct simulations can provide a detailed picture of radial mixing by merely comparing initial and final orbital radii.

What determines planetary spin? Six of our planets have spin vectors aligned with the common orbital vector, while the remaining three (Venus, Uranus, and Pluto) are retrograde. Direct simulations can track the spin vectors of planetesimals to determine the trends in obliquity and distinguish between models where planets are gradually spun up versus those where a massive late-stage impact dominates (Lissauer and Safronov 1991, Dones and Tremaine 1993, Greenberg et al. 1996). This issue is related to the likelihood of creating Earth's Moon with a large impact (Cameron and Benz 1991, Ida et al. 1997). Note that post-formation torquing by solar tides may also affect planetary obliquities (Quinn et al. 1991).

Why is the asteroid belt so sparse? There is only $3 \times 10^{24} \mathrm{~g}$ of material between 2.1 and $4 \mathrm{AU}$. The size distribution is collisionally evolved and the characteristic relative velocities $\left(\sim 5 \mathrm{~km} \mathrm{~s}^{-1}\right)$ are larger than the escape velocity of even the largest asteroid, Ceres. The blame for thwarting accretion and carving "the gaps" is nearly always attached to Jupiter. The first requires the rapid formation of Jupiter (see above). The latter may face problems with the extent of mass depletion compared to the narrow width of the resonances, unless Jupiter's semimajor axis migrated during its evolution so the narrow resonance zones swept through the belt and ejected sufficient material (Lissauer and Stewart 1993). Direct simulations over long timescales that include giant perturbers may be able to show this process in action. We report on one such simulation in Section 3.2. Another possibility is that large planetesimals scattered by Jupiter (or each other) could clear out smaller bodies in this region (Petit et al. 1998). Numerical simulations may provide further insight into this possibility.

What is the role of orbital migration? Recent discoveries (e.g., Marcy et al. 1998 and references therein) of giant planets in surprisingly small orbits around nearby stars have stimulated an interest in orbital migration (Lin et al. 1996) that may arise from gravitational torques (Goldreich and Tremaine 1980), excitation of spiral density waves in the gaseous disk (Ward 1986, 1997), or preferential scattering of planetesimals (Malhotra 1993, Murray et al. 1998). Jupiter could also drive a one-armed spiral density wave in the planetesimal disk of wavelength $\sim 0.5 \mathrm{AU}$ (for nominal disk parameters) at the $\mathrm{g} 5$ secular resonance near $2 \mathrm{AU}$, creating a spiral wave pattern that rotates on a timescale of $\sim 10^{5} \mathrm{yr}$ (Ward and Hahn 1998). These waves and their relative importance in the heating and long-term stability of the planetesimal disk can be examined with direct simulations. This is one more example of the ability of direct simulations to bypass examining such issues in isolation and instead observe their rich interplay. We report on a preliminary investigation of orbital migration in Section 3.4.

\subsection{Previous Work on Planetesimal Problems}

Terrestrial planet formation is divided into four loosely defined stages (see Lissauer (1993) for a review):

Initial stage. Condensation and growth of grains in the hot nebular disk together with gradual settling to the midplane. Gravitational instability among the grains is resisted owing to continuous stirring by convective and turbulent motions.

Early stage. Growth of grains to kilometer-sized planetesimals via pairwise accretion in the turbulent disk. Planetesimals initially have low eccentricities $(e)$ and inclinations $(i)$ due to gas drag.

Middle stage. Agglomeration of planetesimals by focused merging. Possible runaway accretion and subsequent energy equipartition (dynamical friction) may lead to polarization of the mass distribution: a few large bodies with low $e$ and $i$ in a swarm of smaller planetesimals with high $e$ and $i$.

Late stage. Once runaway accretion has terminated due to lack of slow-moving material, protoplanets gradually evolve into crossing orbits as a result of cumulative gravitational perturbations. This leads to radial mixing and giant impacts until only a few survivors remain, over timescales of $\sim 10^{8} \mathrm{yr}$.

At the moment, we cannot unambiguously order the dominant physical mechanisms in the Initial and Early stages. Hence, we are far from being able to embark on a definitive program of simulation in this area. These states will likely be best modeled with the microphysics of electrostatic interaction and gas drag parameterized below the grid used to follow the fluid motions.

Three approaches have been taken toward modeling the Middle to Late stages of planet formation: analytical, statistical, and direct simulation. Safronov (1969) pioneered this with "particlein-a-box" methods that use kinetic theory with collision and scattering operators to evolve the RMS planetesimal velocities (see also Greenberg et al. 1978; Stewart and Wetherill 1988; Wetherill and Stewart 1989, 1993; Palmer et al. 1993). Monte Carlo techniques for the Late stage test for (rare) close encounters between planetesimals and alter their mean motions using a two-body gravitational scattering formula (Wetherill 1990). Statistical techniques often ignore interactions outside the Hill 
TABLE I

Highlights of Advances in D irect Simulations of the Formation of the Inner Planets

\begin{tabular}{lcccccc}
\hline \multicolumn{1}{c}{ Paper } & $N$ & $t(\mathrm{yr})$ & $D$ & $\Delta a(\mathrm{AU})$ & Collisions & Giants? \\
\hline Lecar and Aarseth (1986) & 200 & $6 \times 10^{4}$ & 2 & $0.5-1.5$ & $\mathrm{a}$ & no \\
Beaugé and Aarseth (1990) & 200 & $6 \times 10^{5}$ & 2 & $0.6-1.6$ & abcf & no \\
Ida and Makino (1992a,b, 1993) & 800 & 5000 & 3 & 0.3 & - & no \\
Aarseth et al. (1993) & 400 & $1.2 \times 10^{4}$ & 3 & 0.04 & ab & no \\
Kokubo and Ida (1995, 1996, 1998) & 5000 & $2 \times 10^{4}$ & 3 & 0.4 & a & no \\
Chambers and Wetherill (1998) & 100 & $10^{8}$ & 3 & $0.5-2.0$ & a & yes \\
Richardson et al. $(1998 b)$ & $10^{5}$ & 1000 & 3 & $1.2-3.6$ & a & yes \\
Model B (Section 3.2) & $10^{6}$ & 1000 & 3 & $0.8-3.8$ & a & yes \\
\hline
\end{tabular}

sphere, ${ }^{1}$ so potentially important long-term perturbations are lost (Wisdom 1980, Duncan et al. 1989). Examining the coagulation equation, Wetherill and Stewart (1989) proposed that runaway growth results from dynamical friction. By contrast, Lissauer (1987) asserted that runaway growth ceases when the feeding zone is depleted of slow-moving bodies within a few Hill radii. The protoplanet feeding zones may be replenished by radial migration caused by gas drag on fragmented particles (Wetherill and Stewart 1993), spiral density waves in the gaseous disk (Ward 1986, 1997), or long range perturbative forces between protoplanets (Chambers et al. 1996).

Table I shows the slow evolution of particle number and integration times over the past 12 years for direct simulations (in which all particles contribute to the gravitational potential). Also listed are the parameters from our biggest run so far ( $c f$. Section 3.2). In the table, $N$ is the maximum number of planetesimals used in the simulation, $t$ is the longest integration time, $D$ is the number of spatial dimensions, and $\Delta a$ is either the width of the simulation region at $1 \mathrm{AU}$ or the actual range in orbital distance. If collisions are included in the simulations, details are noted by $\mathrm{a}=$ agglomeration; $\mathrm{b}=$ bouncing; $\mathrm{c}=$ cratering; $\mathrm{f}=$ fragmentation. The final column shows whether perturbations from one or more giant planets are included.

The first direct simulations followed 200 lunar-sized bodies during the Late stage of planet formation in the inner Solar System for an integration time of $10^{4.8} \mathrm{yr}$ (Lecar and Aarseth 1986). Even with just 200 particles, binning techniques were employed to limit the number used to determine gravitational perturbations until the total number of particles became sufficiently small. Later work by Beaugé and Aarseth (1990) added a more realistic collision model from Greenberg et al. (1978). The collective interactions between all planetesimals were finally included about six years ago. Ida and Makino (1992a) verified the Rayleigh form of the $e$ and $i$ distributions used in particle-in-a-box methods while Ida and Makino (1992b) demonstrated dynamical friction between planetesimals of different size. Aarseth et al. (1993)

\footnotetext{
${ }^{1}$ The Hill sphere defines the protoplanet's radius of gravitational influence. It is equivalent to the protoplanet's Roche radius in the Sun's external field; the Hill radii of the present-day planets are given by $10^{-2} a\left(M_{\mathrm{P}} / M_{\oplus}\right)^{1 / 3} \mathrm{AU}$, where $M_{\mathrm{P}}$ is the planet's mass and $a$ is the semimajor axis of the orbit.
}

confirmed that dynamical friction leads to energy equipartition and runaway growth on timescales of $10^{5} \mathrm{yr}$ at 1 AU by modeling a "patch" of the planetesimal disk as a shearing sheet. Ida and Makino (1993) demonstrated that runaway growth is slowed when the planetesimals are heated by an embedded protoplanet. More recently, Kokubo and Ida $(1996,1998)$ used the Hermite integrator (Makino and Aarseth 1992) on the special-purpose computer HARP-2 to simulate up to 5000 planetesimals with embedded protoplanets in a narrow ring centered at $1 \mathrm{AU}$. They found that following a rapid runaway growth stage, the largest protoplanets slow their growth rate and they all grow oligarchically, keeping their orbital separations typically within 10 Hill radii (note our present terrestrial planets are 50-100 Hill radii apart). Tanaka and Ida (1997) have investigated gas drag and found it effective at trapping small planetesimals in the feeding zone. Finally, Chambers and Wetherill $(1996,1998)$ used the mixed-variable symplectic (MVS) integrator of Levison and Duncan (1994) to simulate the Late stage with only a few hundred protoplanets, but integrating for $10^{8} \mathrm{yr}$ and including the giant planets. They found the evolution in each of their simulations to be highly stochastic and noticeably dependent on the perturbations from the giant planets.

Analytical and statistical methods provide an important benchmark for the believability of numerical results, but numerical simulation allows us to replace assumptions regarding planetesimal interactions with the exact laws of Newtonian mechanics. Conversely, numerical results can be used to test the applicability of these indirect methods and guide their improvement as tools to be used to answer specific questions in a timely manner. Some models combine direct and statistical methods, such as the work by Jewell and Alexander (1996) where the protoplanets are followed with a direct method and the field planetesimals are treated statistically. This may allow direct methods to explore even earlier stages of planet growth.

Since our primary goal is to understand the final state of the Solar System, why not ignore the earlier stages and just simulate the Late stage? While Chambers and Wetherill (1998) have shown that this stage can be simulated directly, the initial conditions are a product of the highly nonlinear evolution in the Middle stage. If we do not understand where to lay down a few hundred bodies of lunar size, then we do not know what 
to make of the final state. However, with our code, we can create initial conditions for the Middle stage that are realistic and characterized by global parameters such as the surface density distribution and the properties of the giant planets. With enough particles, we become relatively insensitive to the initial distribution of inclinations, eccentricities, and planetesimal masses. These should evolve quickly to representative states where one can follow the nonlinear dynamics of planetary buildup and disk cleansing. We hope that the system evolves through a generic intermediate state that can be statistically sampled, but we do not know what those generic states are nor even if it requires $10^{6}$ or $10^{3}$ particles to capture them. With our algorithmic advances, we hope ultimately to follow the entire Middle and Late stages with direct simulations.

\section{METHODS}

The simulations described here were performed using a modified version of a cosmological $N$-body code, pkdgrav (Stadel and Quinn, in preparation; data structures described in Anderson 1993, 1996). This is a scalable, parallel tree code designed for ease of portability and extensibility. Load balancing among processors is achieved through domain decomposition: each processor works on particles within a subvolume. These subvolumes are adjusted each timestep according to the amount of work done in the previous force evaluation.

\subsection{Choice of Integrator}

The equations of motion in our simulations are integrated using a leapfrog integrator. Leapfrog has several advantages over other methods:

1. For second-order accuracy, only one force evaluation and one copy of the physical state of the system are required. This is particularly beneficial for $N$-body simulations where the cost of a force evaluation is very expensive.

2. The force field in an $N$-body simulation is not very smooth, so higher order does not necessarily mean higher accuracy.

3. For fixed timesteps, it is a symplectic integrator; i.e., it preserves properties specific to Hamiltonian systems. In the absence of collisions, a planetesimal system is Hamiltonian, and therefore should benefit from the use of an integrator that conserves phase space volume and has no spurious dissipation.

The symplectic nature of leapfrog is easily proven using the operator splitting technique (see Saha and Tremaine 1992 for details; also Yoshida 1990 and Widsom and Holman 1991). In this formalism, the action of the kinetic energy part of the Hamiltonian is described by a drift step-the velocities remain fixed and the positions are drifted forward-while the action of the potential energy is described by a kick step-the positions are held constant and the velocities are kicked according to the acceleration.

Using this terminology, we can describe two variants on leapfrog: (1) drift half a timestep, kick a full timestep, drift half a timestep ("DKD") or (2) kick half a timestep, drift a full timestep, kick half a timestep ("KDK"). These variants are equivalent in terms of accuracy, but the latter is more efficient within a multistepping code, and we use it in the simulations described below.

Note that use of the tree code does not introduce spurious dissipation even though the forces are only approximate. This is because the forces are strictly a function of position, guaranteeing Hamiltonian separability.

\subsection{Spatial and Temporal Adaptivity}

The hierarchical structure of a tree code allows us to follow extremely large dynamic ranges in densities at modest additional cost per force evaluation (e.g., Barnes and Hut 1986, Richardson 1993). However, large ranges in densities also imply a large range in timescales $(\propto 1 / \sqrt{\text { density }})$. Therefore, implementing a scheme such that the force on a given particle is evaluated at a frequency corresponding to its dynamical time can reduce the computational cost significantly.

In pkdgrav, particle timesteps can be chosen to be a powerof-two subdivision (called a "rung") of the basic timestep. This scheme ensures that the particles are synchronized at the end of the basic timestep (see Quinn et al. 2000 for details). It is important to note that in general a variable timestep scheme will destroy the symplectic nature of the integrator. Artificial dissipation can be reduced in variable timestep integrators ( $c f$. Hut et al. 1995, Quinn et al. 2000), but this was not done for the one model we present that used variable timesteps (Model C). This is justified because of the short timescale of the simulation; longer integrations will require this issue to be addressed. We note that there was no evidence of orbital decay among planetesimals in Model C, which would be the telltale sign of artificial dissipation.

In a planetesimal simulation, a logical choice for a timestep criterion is

$$
\Delta t=\eta \sqrt{\frac{r}{\mathcal{F}}}
$$

where $\mathcal{F}$ is the acceleration of the particle, $r$ is the distance, either to the Sun or to the particle that contributes the largest acceleration, and $\eta$ is a dimensionless constant. This criterion has the desirable property that in the absence of interplanetesimal interactions, the planetesimals will have a fixed number of timesteps per orbital period $(n=2 \pi / \eta)$. Other criteria have this property, but they also have drawbacks. For example, a criterion with $\Delta t \propto r / v$, where $v$ is the particle's velocity, is not Galilean invariant. A criterion of $\Delta t \propto \mathcal{F} / \dot{\mathcal{F}}$, would be suitable, but calculating $\dot{\mathcal{F}}$ proved to be computationally expensive.

\subsection{Collision Detection and Resolution}

Collisions are predicted at the beginning of each drift step by keeping the particle velocities fixed and extrapolating the particle positions. Since this is a linear transformation, the time to surface contact between a pair of approaching particles (i.e., for which $\mathbf{r} \cdot \mathbf{v}<0$, where $\mathbf{r}$ and $\mathbf{v}$ are the relative position and 
velocity) is given by

$$
t_{\mathrm{coll}}=-\frac{(\mathbf{r} \cdot \mathbf{v})}{v^{2}}\left\{1 \pm \sqrt{1-\left[\frac{r^{2}-\left(R_{1}+R_{2}\right)^{2}}{(\mathbf{r} \cdot \mathbf{v})^{2}}\right] v^{2}}\right\},
$$

where $R_{1}$ and $R_{2}$ are the physical radii of the two particles. The sign ambiguity is resolved by choosing the smallest positive value of $t_{\text {coll }}$. For any given particle, $N_{s}$ nearest neighbors are considered (typically $8 \lesssim N_{s} \lesssim 32$ ). The neighbors are found in order $N_{s} \log N$ time using a balanced $k$-d tree, which is slightly different than the tree used by the gravity solver. The neighbor search algorithm is described in Bentley and Friedman (1979). If a value of $t_{\text {coll }}$ is found that is less than the size of the drift step, then a collision must be performed. If more than one pair of particles satisfies this condition, the pair with the smallest $t_{\text {coll }}$ value is processed first.

To determine the collision outcome, the relative speed is compared to the mutual escape speed

$$
v_{\mathrm{esc}}=\sqrt{\frac{2 G M}{R_{1}+R_{2}}},
$$

where $M \equiv m_{1}+m_{2}$ is the sum of the particle masses and $G$ is the gravitational constant. If the relative speed is less than the mutual escape speed, the particles are merged to form a new (spherical) object with the same bulk density. Otherwise the particles are allowed to bounce, with some energy dissipation parameterized by coefficients of restitution $\epsilon_{n}$ and surface friction $\epsilon_{t}$ (Richardson 1994, 1995). At higher impact energies, cratering and fragmentation would be expected to take place; we do not model these effects currently but plan to add them in the future (Section 4.1). However, merged particles are checked to ensure that their post-collision angular speeds do not exceed the classical breakup limit

$$
\omega_{\max }=\sqrt{\frac{G M}{R^{3}}},
$$

where $R$ is the radius of the newly merged body. Otherwise the particles involved are forced to bounce off rather than merge. This prevents unrealistic mergers resulting from grazing collisions.

Once the collision outcome has been determined and new velocities have been calculated (either for the two rebounding particles or for the single merged body), the post-collision particles are traced back to the start of the drift step so that they can be included in any remaining collision checks. This ensures that all collisions are detected and treated in the correct order, even if particles are involved in more than one collision during the drift step.

\subsection{Initial Conditions and Analysis}

In order to keep the main code as general as possible, generation of initial conditions and analysis of output are performed using auxiliary programs. Parameters for the initial conditions generator include the number, total mass, and bulk density of the planetesimals, the inner and outer orbital boundaries, the exponent of the surface density power law distribution, the eccentricity and inclination distribution functions (either Rayleigh or Gaussian), the size, location, and gap width of any seed mass, and the number of giant planets to include. An iterative method is used to ensure that particles' Hill spheres do not overlap initially. Parameters used in the integration code itself include the timestep interval (including $\eta$ and the number of rungs if multistepping), the opening angle criterion for the tree (typically between 0.5 and $0.8 \mathrm{rad}$ ), and the values of $N_{s}, \epsilon_{n}$, and $\epsilon_{t}$ (Section 2.3).

Analysis of our simulation output is completely automated and includes plots of the time evolution of all key indicators, such as the number of particles, maximum and average planetesimal masses, and velocity dispersion, and snapshots at arbitrary times of the following: the surface mass distribution; histograms of $e, i$, and mass; $e, i$, and particle spins as a function of semimajor axis and particle mass; and various diagnostic quantities such as energy drift and evaluation times. Our analysis code also includes methods of tracking merger histories and animating time-varying quantities. In addition, the data visualization package $t i p s y^{2}$ that was developed for cosmological simulations with pkdgrav can be used to explore the large data sets.

\section{RESULTS}

Although our code is still under development, we can already begin to address some of the fundamental issues outlined in Section 1.1. Below we present a series of experiments designed both to test and to demonstrate our code and to determine what needs to be improved. Each experiment is briefly introduced, then the results are presented and discussed. Note that for the simulations presented here, there were no boundary conditions on the simulation regions-particles were free to move wherever they liked.

\subsection{Model A: Oligarchic Growth}

Kokubo and Ida (1998) presented some of the highest resolution direct $N$-body simulations of planetesimal dynamics published to date. As a test of our code, we attempted to reproduce their "model (ii)" results which demonstrate oligarchic growth among protoplanets. We used identical starting conditions, namely 4000 equal-mass bodies (mass $3.0 \times 10^{23} \mathrm{~g}$, bulk density $2 \mathrm{~g} \mathrm{~cm}^{-3}$ ) distributed uniformly in a thin annulus of width $\Delta a=0.085 \mathrm{AU}$ centered on $1 \mathrm{AU}$. In addition, the initial eccentricities and inclinations were taken from a Rayleigh distribution with $\left\langle e^{2}\right\rangle^{1 / 2}=2\left\langle i^{2}\right\rangle^{1 / 2}=4 h / a$, where $h$ is the mutual Hill radius, the particle radii were artificially enhanced by a factor of $f=6$, and all collisions resulted in mergers (perfect accretion). The only meaningful difference between the two

${ }^{2}$ http: //www-hpcc.astro.washington.edu/tools/TIPSY/. 


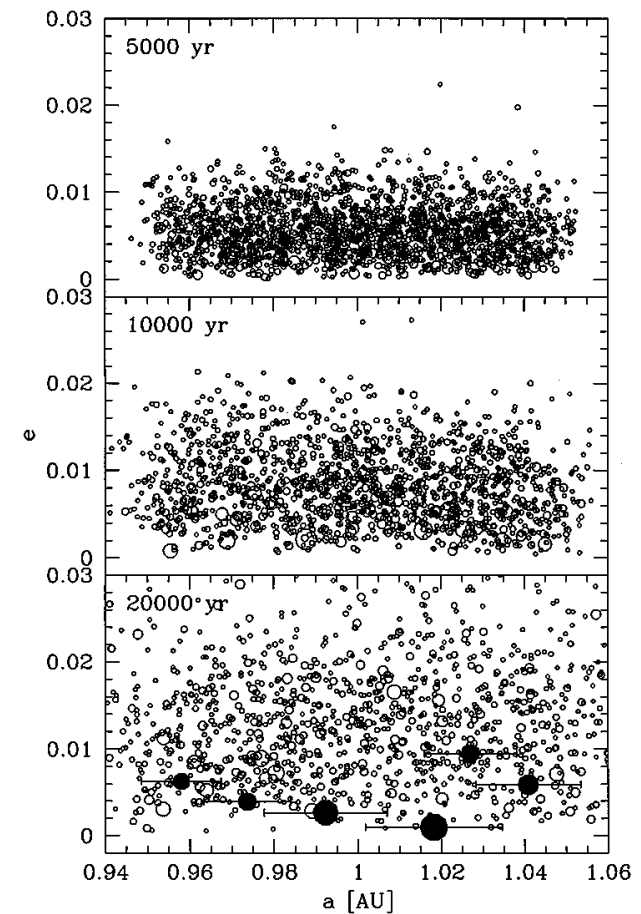

FIG. 1. Three snapshots of semimajor axis $a$ vs eccentricity $e$ for Model A. Circle sizes are proportional to physical radius. In the final frame, the six largest masses are indicated by filled circles with $5 h$ errorbars, where $h$ is the mutual Hill radius. The numbers of planetesimals are $2062(t=5000 \mathrm{yr})$, $1582(t=10,000 \mathrm{yr})$, and $1153(t=20,000 \mathrm{yr})$.

approaches ought to be the method of integration (they used a fourth-order Hermite scheme on special-purpose hardware) and the choice of timestep (they used individual and hierarchical timesteps whereas we chose fixed steps of $0.0025 \mathrm{yr}$ after some experimentation).

Figure 1 shows the time evolution of the planetesimal eccentricity as a function of semimajor axis we obtained for this system (compare with Fig. 4 of Kokubo and Ida 1998). In the bottom frame of the plot, the six largest masses are filled in and their $5 h$ zones are indicated with errorbars. The results are in good agreement with Kokubo and Ida (1998), showing evidence for oligarchic growth of a few protoplanets separated by roughly $5-10$ Hill radii. Note the protoplanet in our simulation with $e \sim 0.1$ will likely be consumed in short order by the nearby most massive protoplanet. The growth of the maximum and mean mass in the system is plotted in Fig. 2. The continued divergence of the maximum mass from the mean indicates that the largest protoplanets are still in the runaway growth phase with respect to the smallest planetesimals. The simulation required 15 wallclock days on a single 433-MHz DEC Alpha PC to complete. Note that this calculation did not take advantage of the parallel processing capability of our code.

\subsection{Model B: Planetesimal Disk}

In order to challenge our code with a fully parallel problem, we elected to expand the previous model to cover a much larger region of the protoplanetary disk. For this test we generated $N=$ $10^{6}$ identical $2 \mathrm{~g} \mathrm{~cm}^{-3}$ planetesimals in a cold $\Sigma(a) \propto a^{-3 / 2}$ disk of total mass $4.7 M_{\oplus}$ that extended from 0.8 to $3.8 \mathrm{AU}$. The present-day outer planets were included in the calculation in order to gauge their effect on planetesimal accumulation. Since the disk started perfectly flat, the mutual inclination of the planets provided a vertical component of acceleration for the planetesimals. No radius scaling was used in this simulation. The run took approximately 200 wallclock hours to complete $1000 \mathrm{yr}$ of integration using a 300-MHz Cray T3E with 128 dedicated processors. Timesteps were fixed at $0.01 \mathrm{yr}$.

Figure 3 shows the evolution in mass density (Figs. 3a-3c) and $a-e / a-i$ distribution (Figs. 3d-3f) of this system. The effect of Jupiter on the disk, which extends well into the present-day asteroid belt, can be clearly seen in the density plots: a large density gap opens up at the 2:1 resonance (3.2 AU) and a narrow groove becomes visible at the $3: 1$ (2.5 AU). Strong transient spiral wave patterns and other telltale features also develop early on before dissolving away. There are corresponding features in the $a-e$ plot which show how Jupiter stirs up planetesimals at the mean-motion resonances. Note that conservation of the Jacobi integral accounts for the bending of the $e$ peaks toward smaller $a$. Effects from the other outer planets are too weak to be seen yet. Meanwhile, planetesimal growth has proceeded uninterrupted in the inner region of the disk (under the assumption of perfect accretion). However, due to the realistic particle sizes the largest planetesimal at the end of the run is only nine times its starting mass. As far as we are aware, this is the largest

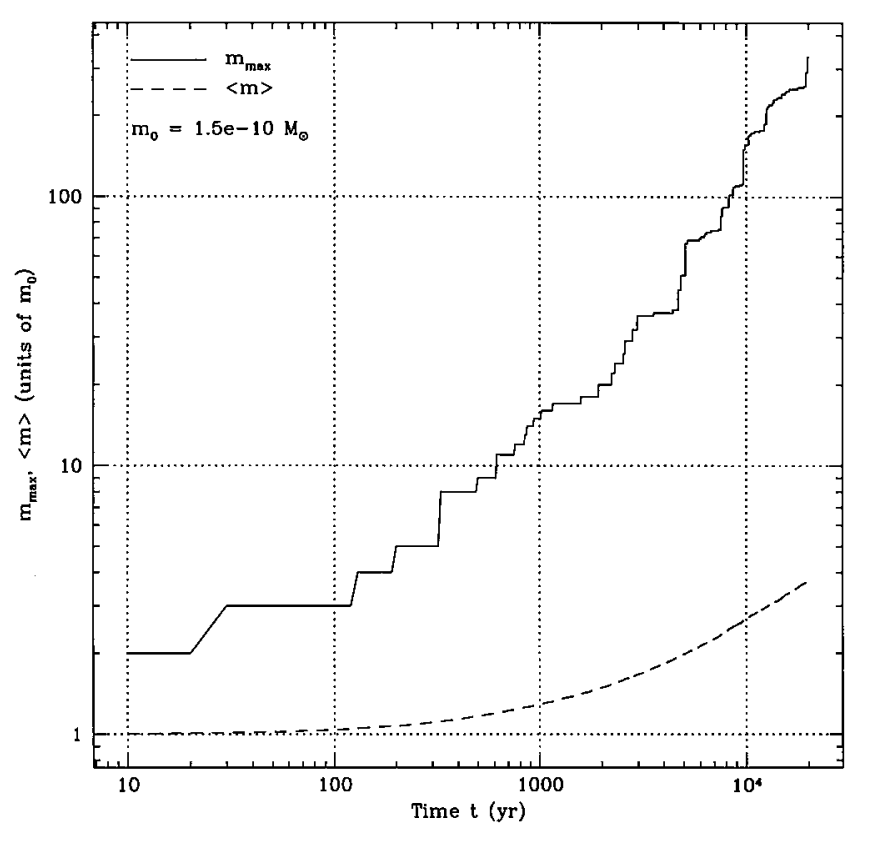

FIG . 2. Evolution of the maximum mass $m_{\max }$ (solid line) and mean mass $\langle m\rangle$ (dashed line) for Model A. At the end of the simulation, $m_{\max } /\langle m\rangle \sim 90$ and the maximum mass is diverging, indicating runaway growth. The other protoplanets are growing at a similar rate with respect to the smaller planetesimals, giving rise to oligarchic growth. 

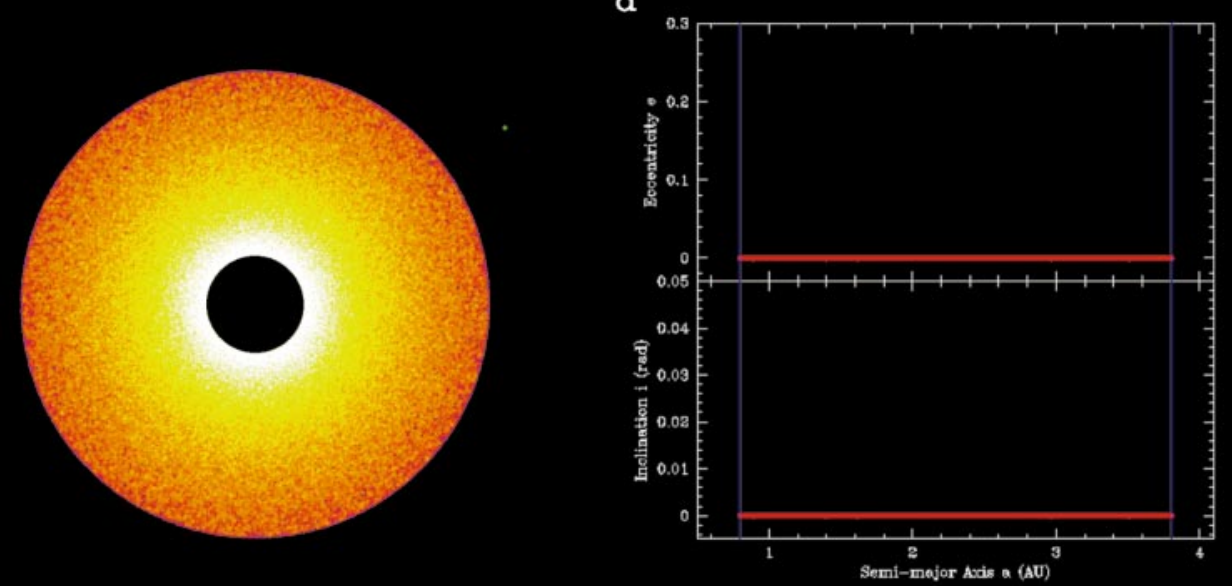

b

e
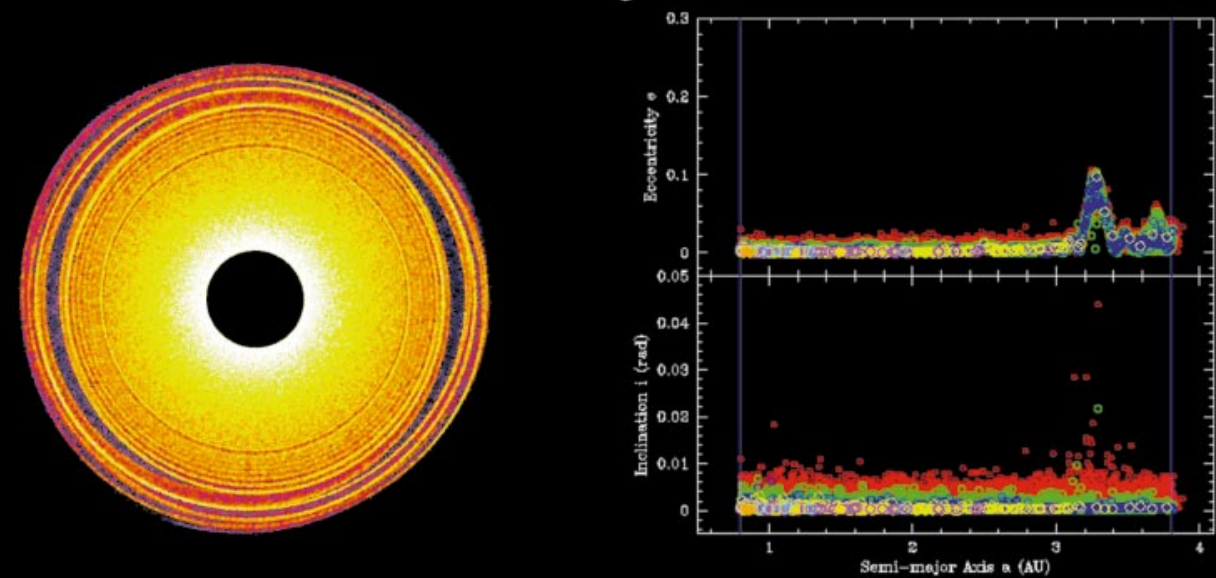

C

$\mathbf{f}$
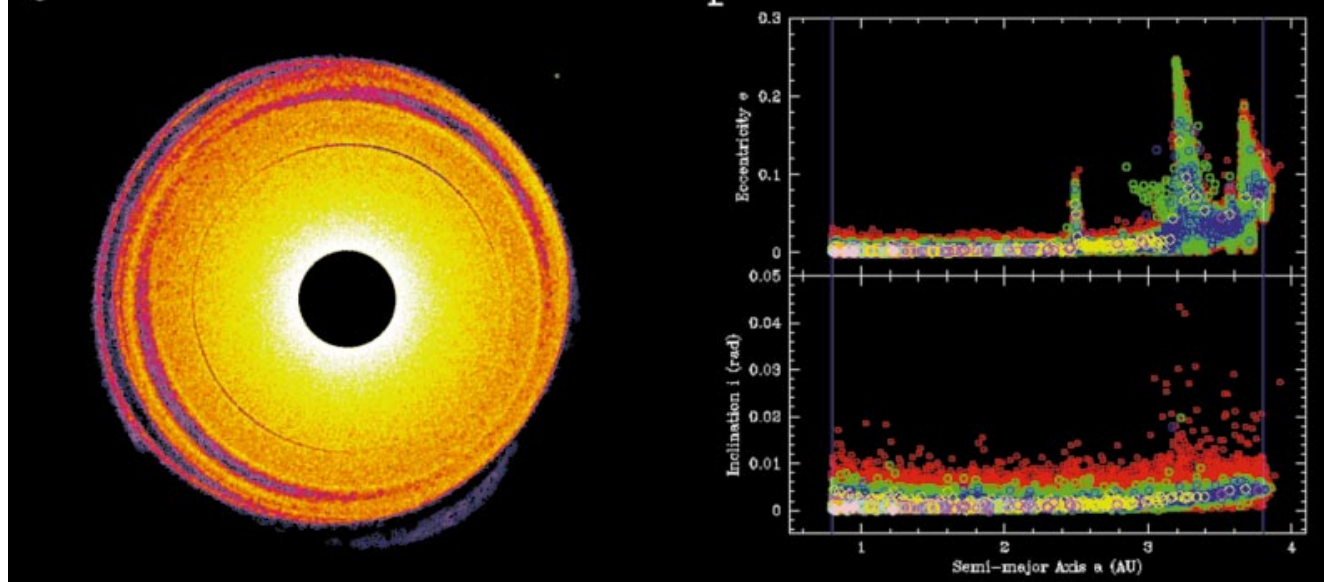

FIG. 3. Sequence showing the evolution of the mass density and $a-e / a-i$ distribution for Model B. Frames (a) and (d) show the initial condition; (b) and (e) show the state after $100 \mathrm{yr}$; and (c) and (f) show the state after $890 \mathrm{yr}$, toward the end of the run. In frames (a) through (c), bright colors indicate regions of high density. The tiny green dot outside the disk marks the location of Jupiter. The sharp inner ring in frames (b) and (c) is the 3:1 resonance; the larger crescent-shape gaps aligned with Jupiter are at the 2:1 resonance. Note the shred of material at the outer edge of the disk in the last frame; this material is at the distance of the 3:2 resonance (at $3.97 \mathrm{AU}$ ), even though the disk originally extended only to $3.8 \mathrm{AU}$. In frames (e) and (f), circle size is proportional to particle radius (as in Fig. 1). The particles are also color-coded by size, with the smallest red circles indicating pristine material, larger green circles indicating bodies that have undergone one collision, blue circles two collisions, and so on (the color sequence repeats at larger masses). The growth of the major mean-motion resonances is clearly seen in frames (e) and (f). The bending of the $e$ peaks to smaller $a$ is a consequence of conservation of the Jacobi integral. Note the cloud of green particles to the left of the 2:1 resonance in the final frame: these particles were presumably ejected from the resonance after undergoing a merger. The material at the outer edge with moderate eccentricity corresponds to the shred in frame (c). The largest masses are concentrated at low $e$ and $i$ toward the inner portion of the disk. 


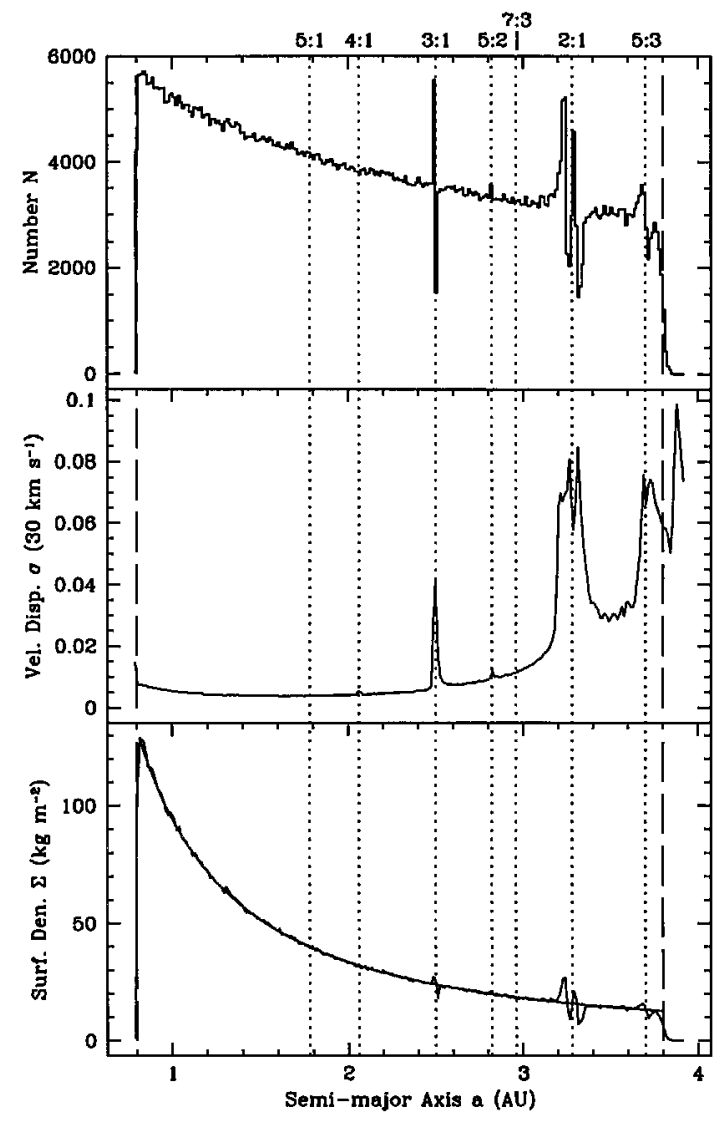

FIG . 4. Three plots showing the histogram of $a$ values (top), the velocity dispersion $\sigma$ as a function of $a$ (middle), and the surface density as a function of $a$ (bottom) near the end of the Model B run. The Jupiter mean-motion resonances are indicated with dotted vertical lines and can be seen to correspond with local features in the plots in most cases. The dashed vertical lines delineate the initial starting region. In the bottom plot, the initial surface density distribution is indicated by a solid curve.

simulation of a self-gravitating planetesimal disk that has ever been attempted.

More indications of the effect of the Jupiter resonances can be seen in Fig. 4, which shows a histogram of planetesimal semimajor axis along with the velocity dispersion and surface density across the disk. The resonances are indicated with dotted vertical lines. Most of the plots show telltale features at all but the weak fourth-order resonances. Note the double peak at the 2:1 resonance, which may indicate the presence of a quasi-stable libration zone, and the sharpness of the 3:1 resonance. Generally particles near the mean-motion resonances are displaced to smaller $a$ due to conservation of the Jacobi integral as the eccentricities are pumped, causing a dip to the right of the resonance line and a peak to the left. We do not see any evidence of the g5 secular resonance mentioned in Section 1.1, but it is still too soon to expect any strong features.

Figure 5 shows the number of mergers as a function of $a$. Initially the merger rate was very large due to the cold start: there were $\sim 60,000$ mergers in the first $50 \mathrm{yr}$, and only $\sim 15,000$ in the remaining $950 \mathrm{yr}$. This may explain why there is little evidence in Fig. 5 of any sharp changes to the merger rates near the Jupiter resonances, since the scattering took a while to develop (Fig. 3). Hence we cannot determine from the basis of our simulation so far whether the presence of the resonances impedes or enhances planetesimal growth.

\subsection{Model C: Galilean Satellites}

Given the long timescales involved in the planetesimal disk simulation, we decided to investigate a system with a more favorable evolution time. Such a system can be found by maximizing the timescale ratio $\sqrt{a^{3} \rho / M_{\mathrm{c}}}$, where $a$ is the orbital distance from a central mass $M_{\mathrm{c}}$ and $\rho$ is the local mass density. For the solid component of the solar nebula at $1 \mathrm{AU}$ in the minimum mass model, this ratio is $\sim 2 \times 10^{-3}$, assuming the disk height varies as $\sim 0.1 a$. For Jupiter's protosatellite disk we estimate the timescale ratio to be $\sim 8 \times 10^{-3}$, assuming the disk extends over the region presently occupied by the jovian moons ( 0.0028 to $0.013 \mathrm{AU})$. Simulations of the formation of the Earth's moon fare even better, with a timescale ratio of $\sim 3 \times 10^{-1}$ (Ida et al. 1997), though this is largely due to the assumption the Moon formed just outside the tidal radius and subsequently migrated outward. Of course, for the jovian moon system, we are assuming gas accretion onto the planet has ceased and will not interfere with the accumulation of the satellites in a significant way. For these tests this simplification is justified.

We ran two test cases: Model $\mathrm{C} 1$ had a surface density distribution law $\Sigma(a) \propto a^{-3 / 2}$; 2 had $\Sigma(a) \propto a^{-1}$. The former distribution is consistent with smearing out the mass of the present jovian moons in the same manner as is conventionally

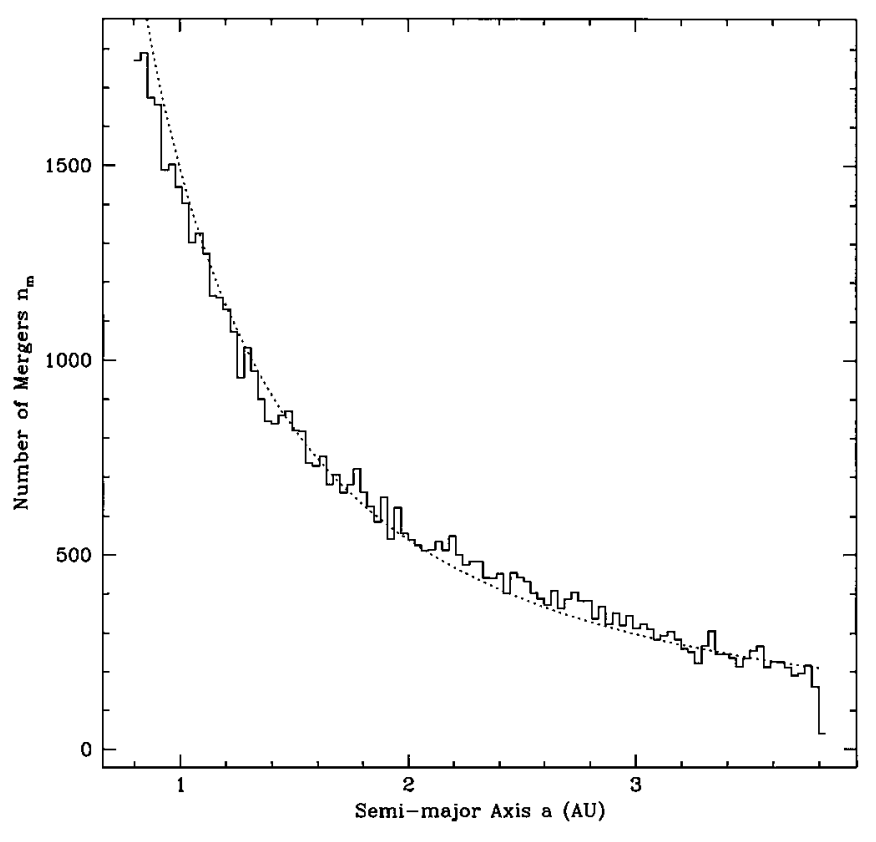

FIG .5. Histogram of the number of mergers as a function of $a$ for Model B. The dotted curve is a power-law fit to the distribution with exponent -1.5 . There is little evidence of local changes to the merger rate near the Jupiter resonances. 
done for the Solar System. The latter distribution was chosen for comparison following recent work by Pollack et al. (1996) and Chambers and Wetherill (1998). The only other difference between the models was that $\mathrm{C} 1$ had $N=25,000$ initially, whereas $\mathrm{C} 2$ had $N=10,000$, as it was run on a slower platform. The total mass in both cases was $0.065 M_{\oplus}$, or roughly twice the mass of the present Galilean satellites. The starting disk extended from 0.002 to $0.015 \mathrm{AU}$ (note the Roche limit for $1 \mathrm{~g} \mathrm{~cm}^{-3}$ particles is $0.0013 \mathrm{AU}$, comfortably inside the inner edge of the adopted disk) and particles were assigned fairly cold Rayleigh distributed eccentricities and inclinations according to $\left\langle e^{2}\right\rangle^{1 / 2}=2\left\langle i^{2}\right\rangle^{1 / 2}=0.2 h / a$. The bulk density of the planetesimals was set to $1 \mathrm{~g} \mathrm{~cm}^{-3}$ to reflect their likely more icy and porous composition. Both models made use of the multistepping facility of the code, with a basic maximum step of 0.01 time units (about $14 \mathrm{~h}$, or one-third of an Io year), eight timestep rungs, and $\eta=0.02 \pi$ (100 steps per orbit in the absence of interplanetesimal perturbations). Throughout the calculation, generally $85 \%$ of the particles occupied the middle rungs while the remaining $15 \%$ occupied the highest rungs, i.e., those with the shortest timesteps. Collision outcomes were governed by the rules outlined in Section 2.3 (i.e., both merging and bouncing were included), with $\epsilon_{n}=0.8$ (mostly elastic, for illustrative purposes) and $\epsilon_{t}=1.0$ (no surface friction).

Figures 6 and 7 show the $a-e / a-i$ distribution and mass histogram, respectively, of Model $\mathrm{C} 1$ at the end of the simulation (after $10^{4}$ maximum steps, i.e., about 16 yr or $\sim 3000$ Io orbits).

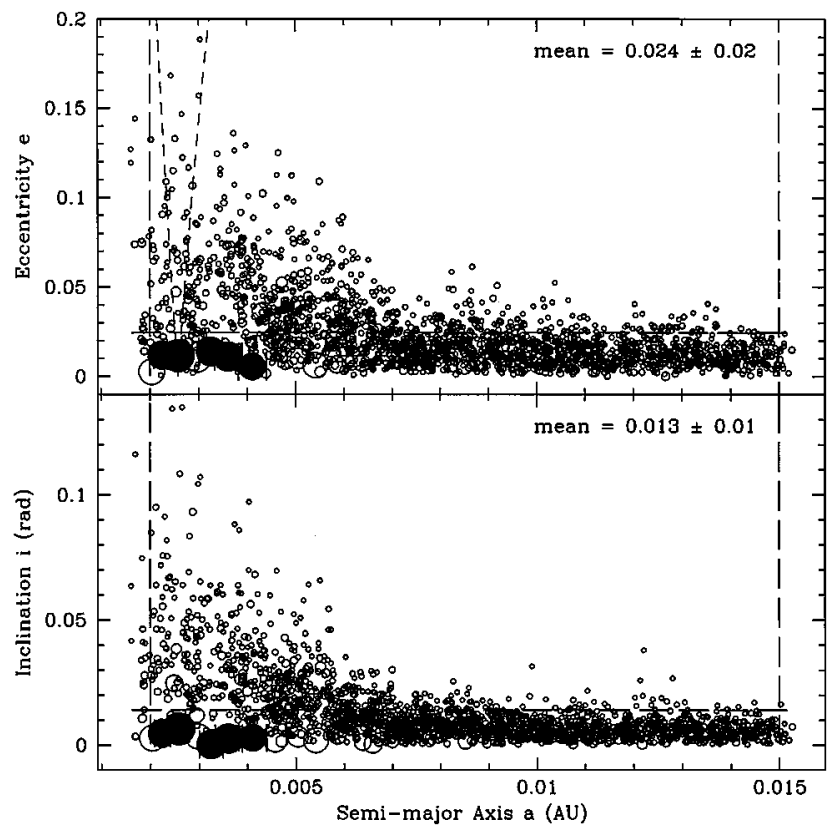

FIG. 6. Distribution of $e$ and $i$ as a function of $a$ at the end of Model C1. Only the largest 2000 particles are shown, to avoid overcrowding the figure. As usual, circle size is proportional to particle radius. The five largest masses are drawn as filled circles, with $5 h$ errorbars. The curved dashed lines are lines of constant periapse and apoapse for the largest mass, indicating the likely trajectory of scattered planetesimals. The horizontal dashed lines indicate mean values.

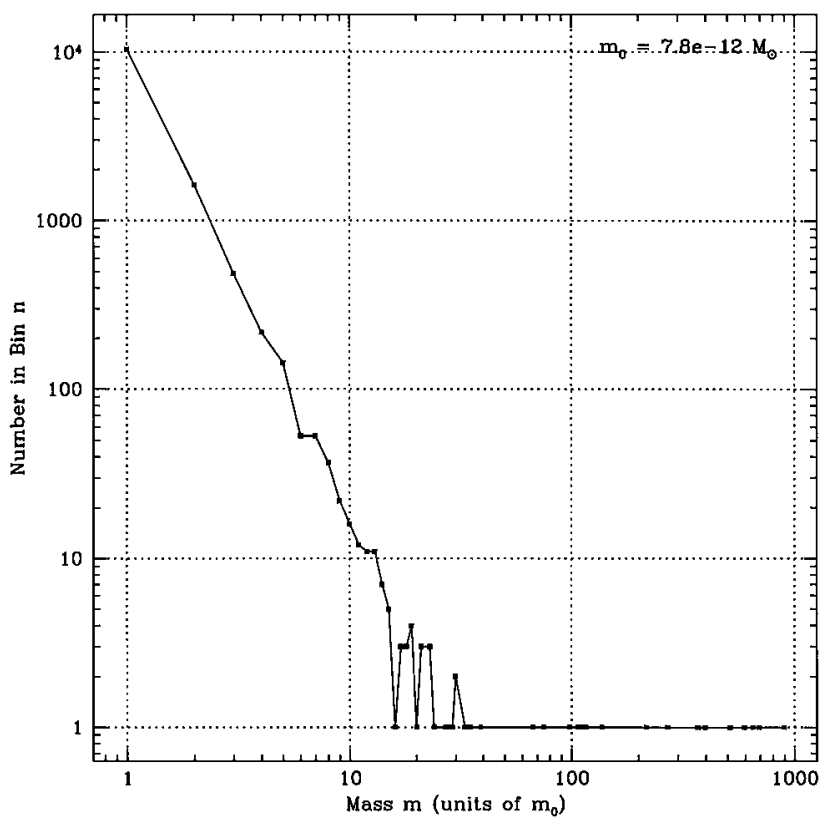

FIG. 7. Mass histogram for Model C1. The power-law slope (for $m \lesssim$ $15 m_{0}$ ) has exponent -2.7 .

Just over 13,000 particles remain at this point (52\%), with the bulk of the material located outside $0.005 \mathrm{AU}$. The largest mass is nearly 1000 times its starting value and there are more than a dozen particles larger than 100 times the starting mass. The slope of the $n$ vs $m$ power law is -2.7 , comparable to values found in similar studies (e.g., Kokubo and Ida 1996). There is no evidence of oligarchic growth yet; the evolution is still at the runaway stage in the inner region. The largest mass has a spin period of $8.7 \mathrm{~h}$ and an obliquity of $91^{\circ}$; i.e., the embryo is lying on its side.

Corresponding plots for Model C2 are shown in Figs. 8 and 9. This run was also terminated after $10^{4}$ maximum steps, at which time there were just over 5900 particles remaining (59\%). In this case the largest masses are only about 100 times their starting value, and it appears the system is beginning to enter a period of oligarchic growth, with the 5 largest bodies separated by more than 5 mutual Hill radii (though note the presence of somewhat smaller but still substantial embryos in the vicinity). The slope of the $n$ vs $m$ power law is -2.9 , indicating more mass in smaller bodies. This probably reflects the fact that the shallower $a^{-1}$ surface density distribution gives rise to a slower accretion rate in the inner regions. The largest mass in this case has a spin period of $5.6 \mathrm{~h}$ and an obliquity of $75^{\circ}$.

Neither model shows clear evidence of the development of anything resembling the present-day Galilean satellite system. At face value the simulations seem to suggest that the largest body in the system ought to be Io (which is in fact the third largest of the four jovian moons), this despite the criteria imposed for bouncing (rather than merging) which prevent the higher-velocity-dispersion particles in the inner regions from merging straight away. However, the systems clearly are still in 


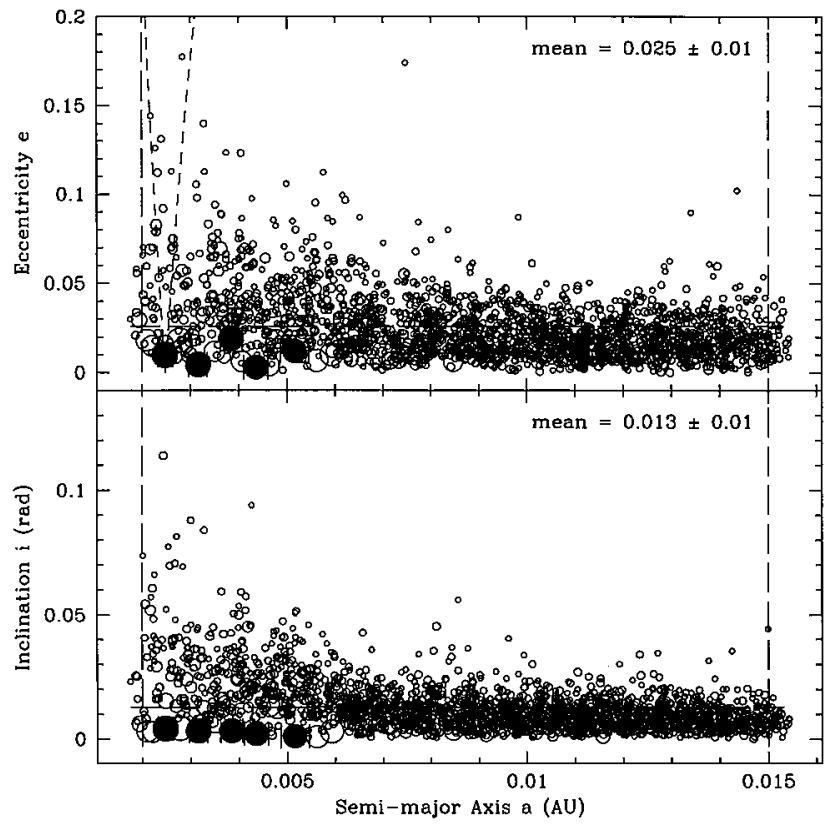

FIG. 8. Same as described in the legend to Fig. 6 but for Model C2, which had a shallower initial surface density distribution and fewer particles.

the initial growth stage; the bulk of the mass is located in the exterior regions where the accretion time is slowest. Moreover, compared with the Moon formation experiments of Ida et al. (1997), we expect the final development of the moons to take at least $5 \times 10^{4}$ dynamical times, a factor of 10 more than achieved so far. Model $\mathrm{C} 1$ required approximately 10 wallclock days on a four-processor 180-MHz SGI Origin 200; Model C2 needed

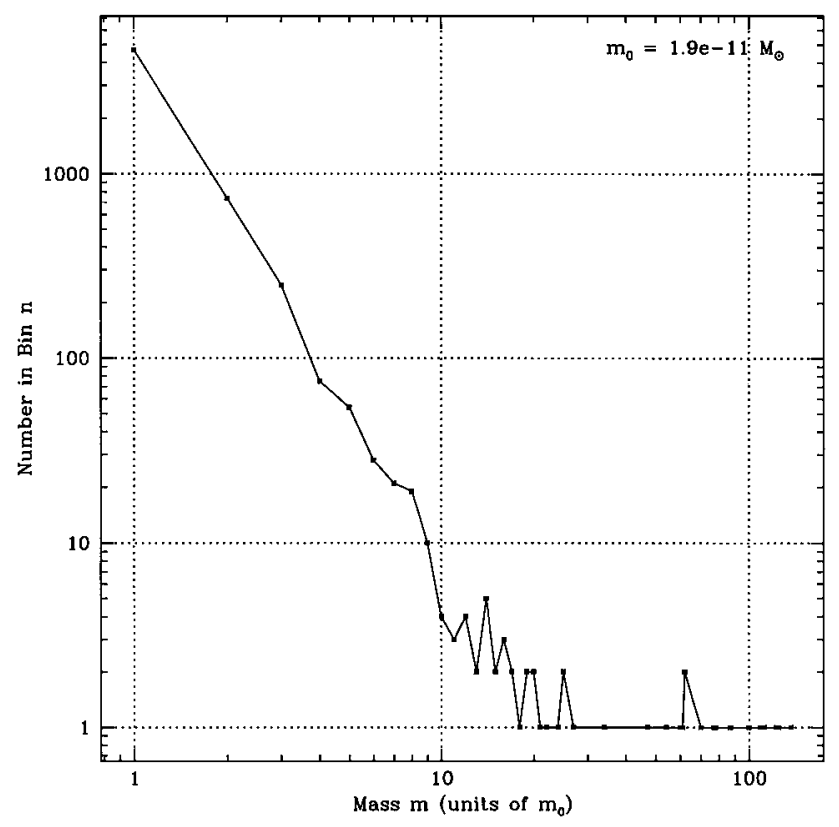

FIG. 9. Mass histogram for Model C2. The power-law slope (for $m \lesssim$ $10 m_{0}$ ) has exponent -2.9 .
8.5 wallclock days on a slower four-processor $200-\mathrm{MHz}$ Intel Pentium Pro. Hence to get to the point of final accumulation in a practical amount of time, either a more powerful platform or a better optimized integration scheme ( $c f$. Section 4.1) is required.

\subsection{Model D: Orbital Migration}

One type of orbital migration that may be amenable to direct simulation arises from planetestimal scattering (Malhotra 1993, Murray et al. 1998). In this scenario, planetesimals lose angular momentum through resonant interactions with a planet embedded in the disk. The eccentricities of the perturbed planetesimals increase until they either collide with the planet or are ejected, thereby removing energy from the planet's orbit and causing the planet to migrate inward. Outward migration is possible if a significant fraction of the perturbed planetesimals collide with the central star (or, in the case of multiple planets, if planetesimals are passed inward from planet to planet until an ejection occurs; $c f$. Malhotra 1993). Due to the large number of particles required to simulate this process and the long timescales involved, this problem has been out of reach of direct methods. Although we cannot yet treat the timescale issue, we are nonetheless at a stage where we can begin to study the dynamical effects of a planet embedded in a disk in a self-consistent fashion at high resolution.

We ran two models to investigate orbital migration. The models differed only in the total mass of the disk and the computational platform used to run the models. In the first model, D1, the total disk mass was set to $1000 M_{\oplus}$, or about three times the mass of the embedded Jupiter-size planet. Such a large disk mass was chosen to ensure that the local surface density would exceed the minimum value required for significant migration (Murray et al. 1998). In model D2, the disk mass was $2000 M_{\oplus}$. In both models the disk extended from 0.5 to $3.5 \mathrm{AU}$ with the usual $a^{-3 / 2}$ law, and the seed mass was located at 2.0 AU. A gap of half-width $0.42 \mathrm{AU}$ was left empty around the seed, corresponding to the "chaotic zone" of the planet (Wisdom 1980). The planet initially had zero eccentricity and inclination while the planetsimals had the same initial dispersions as for Model C. Planetesimals were allowed to merge on contact, but not bounce (the bulk density of the planetesimals was $2 \mathrm{~g} \mathrm{~cm}^{-3}$ ). The timesteps were fixed at 0.01 yr. Both models began with $N=10^{5}$ planetesimals.

Model D1 completed 13,600 steps in 10 days on a cluster of four 433-MHz DEC Alpha PCs connected with a fast ethernet switch. Model D2 required just five days to complete 12,600 steps on a cluster of 14 300-MHz Intel Pentium IIs, also wired together with a fast ethernet switch. However, both runs exhibited periodic sharp rises in CPU usage per step until it was no longer practical to keep the simulations running. We believe this was caused by the excessive computational demands of keeping escaping particles inside the tree structure. At the end of Model D1, 245 particles had $e>1$ with respect to the Sun and the largest value of $a$ was 1700 AU. For Model D2, 330 particles had $e>1$ and the largest value of $a$ was 4200 AU. One particle in each run made it out as far as 10,000 AU. This indicates that particles on escape trajectories should simply be removed 


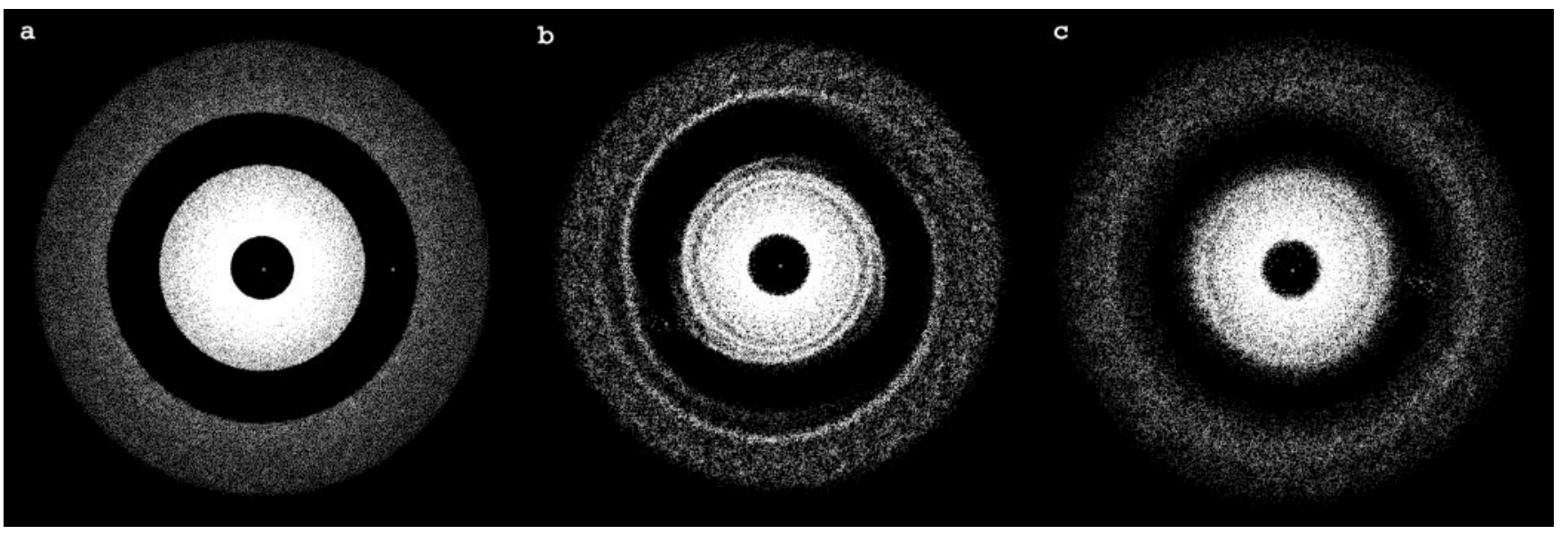

FIG . 10. Sequence showing the evolution of Model D2. Bright regions correspond to high density. (a) is the initial condition, (b) is after 10 yr and shows the strong transients as the disk reacts to the seed; (c) is the final state, after $126 \mathrm{yr}$. The large dot at the center is the Sun and the large dot in the gap is the seed. Note the particles in the immediate vicinity of the seed and the Sun: they will likely be ejected from the system.

from the simulation in future runs. In both runs only one collision with the Sun was recorded. There were 71 collisions with Jupiter in Model D1, and 78 in Model D2. In both cases about $10 \%$ of the particles had merged with each other by the end of the simulation, and the largest mass was 12 times the initial value.

Figures 10-13 show results from Model D2. The plots for Model D1 do not differ significantly from D2 so they are not shown here. Figure 10 is an evolutionary sequence showing the initial development of spiral density waves in the disk due

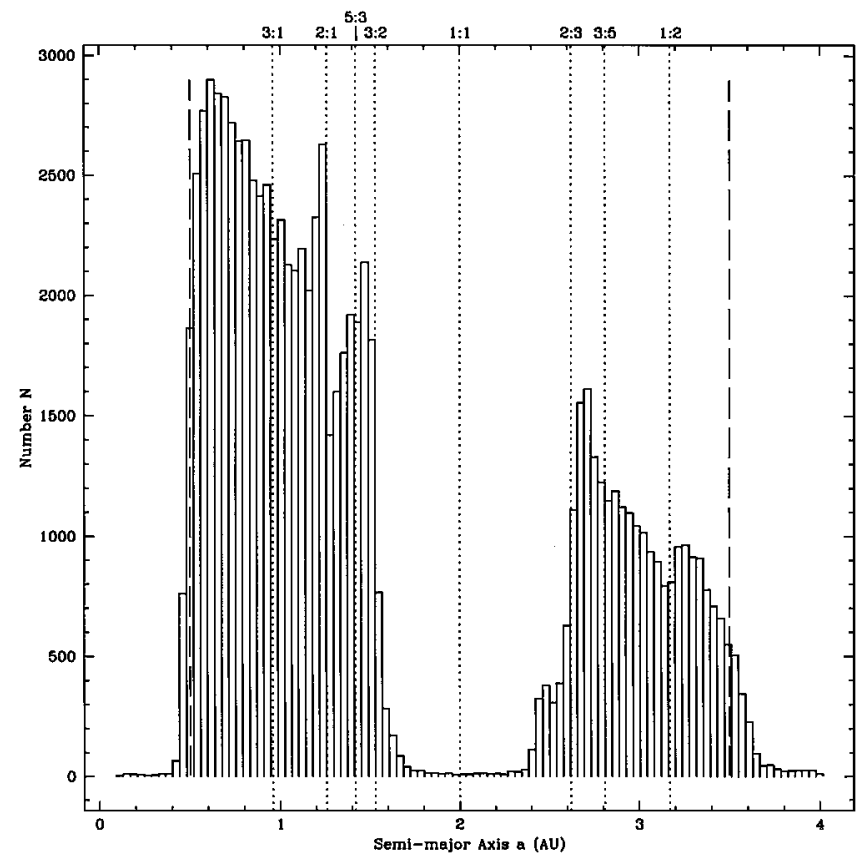

FIG. 11. Histogram of $a$ for Model D2. The large gap in the center corresponds to the chaos zone of the seed particle and was initially void of planetesimals. to perturbations from the seed, and the final quasi-equilibrium configuration with density spikes and troughs near the low-order mean motion resonances. Figure 11 is a histogram of planetesimal semimajor axes in the vicinity of the starting disk. The lowest order resonances are indicated with dotted vertical lines. With the exception of the 3:2 and 2:3 resonances, which are on the edge of the initial gap, each resonance corresponds to a local feature in the histogram ( $c f$. Section 3.2). Similar features can be seen in Fig. 12, which plots the local surface density in the disk. The smooth curve shows the form of the initial power-law distribution for comparison. The outer resonances are not as easily

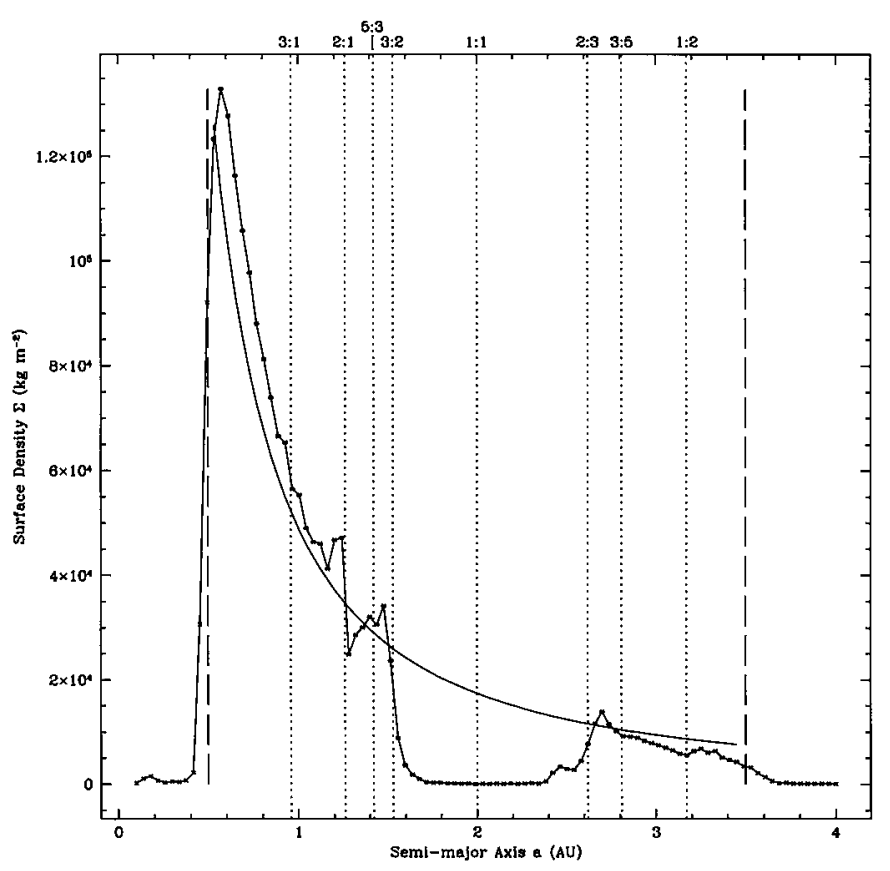

FIG. 12. $\Sigma(a)$ for Model D2. The smooth curve is the original power-law distribution. 


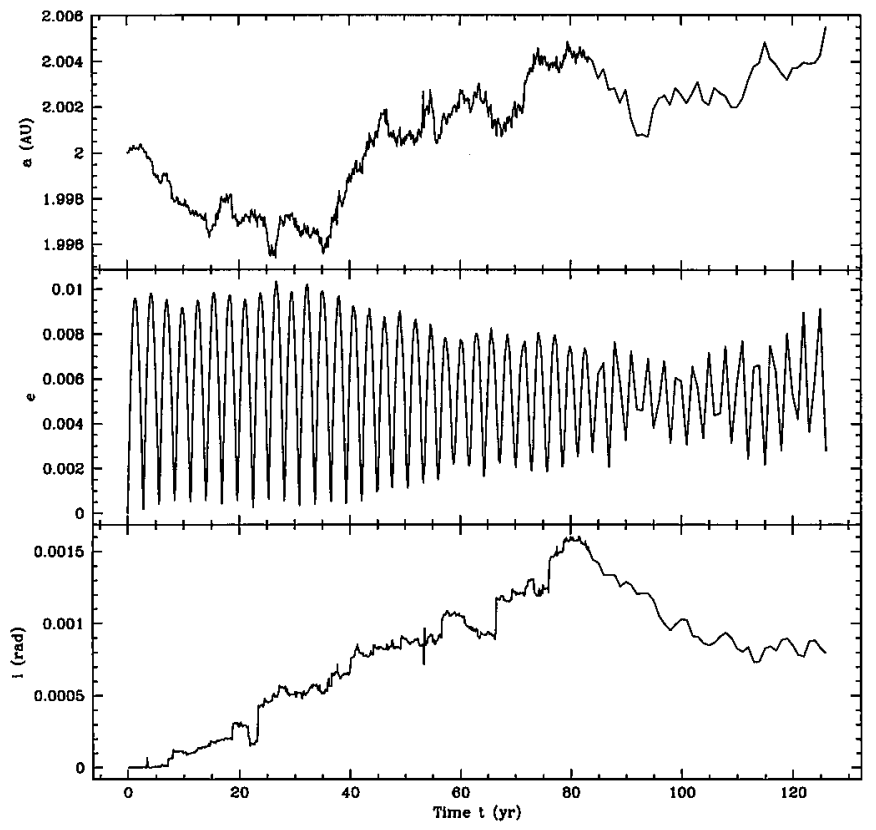

FIG. 13. Plots of the $a$ (top), $e$ (middle), and $i$ (bottom) evolution of the seed mass in Model D2. The sampling interval was increased from 0.1 to $1 \mathrm{yr}$ around $85 \mathrm{yr}$. It is too early to determine whether there is a clear secular trend in any of the elements.

seen in this plot, due to the relatively low surface density in the outer regions.

Finally, Fig. 13 shows the $a, e$, and $i$ evolution of the seed mass (note that the sampling rate was reduced after $85 \mathrm{yr}$ ). Initially the seed moves inward but after $50 \mathrm{yr}$ it moves out, reaching nearly $2.006 \mathrm{AU}$ at the end of the simulation. The total excursion was about $0.01 \mathrm{AU}$. However, the evolution is quite stochastic, as evidenced by the large $0.004 \mathrm{AU}$ excursion between 70 and $90 \mathrm{yr}$, so it would be premature to deduce a secular trend from the data. Evidently a longer integration time is needed to resolve this ambiguity. This is not surprising given that a crude linear extrapolation to time $t=0$ of the data presented in Murray et al. (1998) shows a migration rate of only $10^{-7} a \mathrm{AU} \mathrm{yr}^{-1}$ even for their best case. Similarly, it is too early to tell whether there is an increasing trend in the seed eccentricity as would be expected for the chosen mass-to-disk ratio (the rapid oscillation seen in the plot is the 2.8 -yr period corresponding to the seed's mean orbit). Even with the expected gains from the Kepler drift method (Section 4.1) this problem may remain out of reach for direct simulation. However, smaller problems to study limiting or transient behavior in strongly perturbed disks are now feasible with our code.

\section{CONCLUSIONS}

We have described a new direct numerical method for simulating planetestimal dynamics. We have shown that the code is capable of handling data sets as large as $N \sim 10^{6}$ for hundreds of dynamical times. Simple experiments with the code confirm the oligarchic growth mode of planetestimals in the terrestrial region, illustrate the effect of jovian resonances on the inner planet disk, reveal possible runaway and oligarchic growth in the jovian moon system, and demonstrate torquing by a disk on an embedded planet. The next major step is to increase the timescale by several orders of magnitude to extend investigations from initial transient regimes to regimes of longer term perturbations. Preliminary work toward this goal is described in the following section.

\subsection{Future Work}

In order to increase the timescale capabilities of our code, we need to take advantage of the fact that planetesimals spend much of their time only weakly interacting with each other. Away from interactions, the planetestimals can be moved along near-Kepler ellipses modified by the perturbative force of all the other planetesimals. Hence we can divide the Hamiltonian into a Kepler component implemented using Gauss' $f$ and $g$ functions, and a perturbation component owing to the force contributions of all the other particles. In this domain, timesteps can be of order the dynamical (i.e., orbital) time, resulting in computational speedups of $\sim 10-100$. For strongly interacting particles (defined as particles with overlapping Hill spheres), the Hamiltonian can be factored into the standard kinetic and potential energy components, with the central force of the Sun as an external potential. In this domain, the timesteps are short enough that collision detection can approximate the trajectories as small linear segments, as outlined in Section 2.3.

The challenge is to predict when particles change between the regimes of weak and strong interaction. One approach that we are considering is to construct a new binary tree ordered by perihelion $q_{i}$ and aphelion $Q_{i}$. Those particles with $Q_{i}+h_{i}>q_{0}-h_{0}$ and $q_{i}-h_{i}<Q_{0}+h_{0}$, where $h$ is the mutual Hill radius and subscript 0 denotes the reference particle, would be flagged for further testing. This screening has a cost of $N \log N$ and would only be performed once per long Kepler step. Flagged pairs of particles with phases that are certain to stay separated over the integration step would be reset. The remaining (few) particles would be tested by solving Kepler's equation in an elliptical cylindrical coordinate system to determine the time of Hill sphere overlap. Switching between Hamiltonians is not strictly sympletic, but it occurs infrequently enough for any given particle that it should not be a concern. Dissipative collisions are inherently nonsymplectic anyway. Once particles separate beyond their Hill spheres (or merge), they would be returned to the Kepler drift scheme.

We have already implemented and tested the Kepler drift scheme. Figure 14 shows the power spectra of $a^{1 / 2}$ (the action conjugate to the mean anomaly) and the Poincaré variables $h=$ $e \sin \varpi, k=e \cos \varpi, p=\sin (i / 2) \sin \Omega$, and $q=\sin (i / 2) \cos \Omega$, for a $10^{6}$-yr integration of Jupiter using the Kepler drift code in pkdgrav (solid line) and an MVS integrator based on Saha and Tremaine (1992; dashed line). Both calculations included Saturn, Uranus, and Neptune, and used 0.5-yr timesteps with outputs every $10 \mathrm{yr}$. The test shows an excellent match in all 


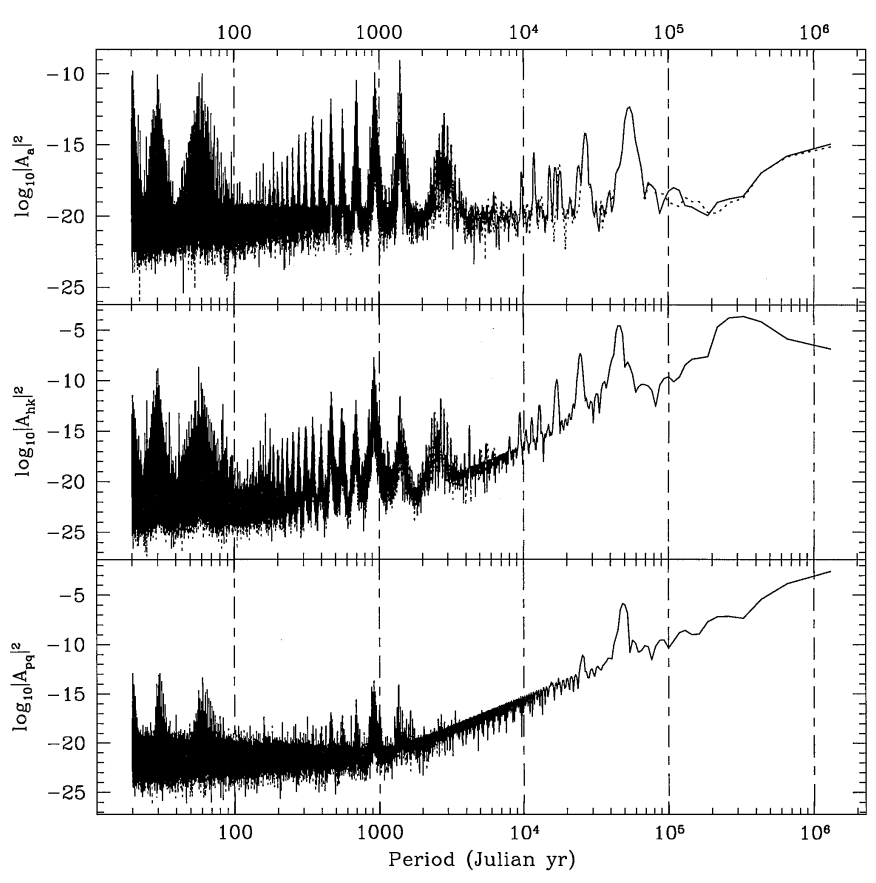

FIG. 14. Power spectra of Jupiter's action conjugate to the mean anomaly $\left(a^{1 / 2}\right)$ and Poincaré variables $h, k, p$, and $q$ for a $10^{6}$-yr integration of the outer giants. The solid line was generated with pkdgrav using the Kepler drift method, the dashed line with Saha and Tremaine's (1992) MVS integrator. The lines are nearly coincident in most cases. Timesteps were $0.5 \mathrm{yr}$ with outputs every $10 \mathrm{yr}$. A Hanning window function was applied to the data. Negative frequencies were used for the $A_{p q}$ plot to bring out the secular resonances (the line of nodes precesses backward). The major resonance features known from previous work (e.g., Applegate et al. 1986) with periods $>10^{3} \mathrm{yr}$ include the g6 secular resonance at $46 \mathrm{kyr}$ in the $A_{h k}$ plot, as well as a blend of g5 and g7 at $300-400 \mathrm{kyr}$ and the linear combination $2 \mathrm{~g} 6-\mathrm{g} 5$ at $25 \mathrm{kyr}$, and the s6 secular resonance at $49 \mathrm{kyr}$ in the $A_{p q}$ plot. The "Great Inequality" at $1.3 \mathrm{kyr}$ can also be seen.

resonance frequencies, indicating that the Kepler drift method is stable over long timescales. It remains to successfully implement the encounter detection algorithm, which is presently our main research focus.

Other areas for future work include implementation of a full collision model that allows for cratering, mass transfer, and fragmentation (Greenberg et al. 1978, Beaugé and Aarseth 1990). The code already supports dynamic creation of particles so this will not involve too much extra work. We currently do not make provision for gas drag, since the planetesimals we model are massive enough to decouple from the nebular gas (Lissauer and Stewart 1993, Ohtsuki et al. 1993). However, once fragmentation is implemented, it will be necessary to include gas drag to ensure a self-consistent treatment of planetesimal dynamics at the low-end mass scale. Other improvements for the future include optimization of the multistepping code and options for initial particle size distributions.

\subsection{Related Applications}

Our technique has important applications in areas beyond those already discussed here. For example, local simulations of Saturn's rings (Salo 1992, Richardson 1994) have shown that transient aggregates and gravitational wakes may form in the dense A and B rings, giving rise to structures on scales of $\sim 100 \mathrm{~m}$. However, these simulations were limited to spatial extents of a few times the instability wavelength, calling their validity into question. With the necessary components for handling collisions properly, our new method could do far better on this problem. It would be a timely project as well: existing images of Saturn's rings are not detailed enough to resolve the wakes and aggregates, but the upcoming Cassini mission to Saturn promises resolutions as good as $\sim 50 \mathrm{~m}$ in the outer rings and perhaps $\sim 150 \mathrm{~m}$ in the B ring (L. Dones 1998, personal communication).

Our code can also be used to follow the tidal disruption of "rubble piles" (loose aggregations of material held together solely by self-gravity). The spectacular breakup of Comet Shoemaker-Levy 9 at Jupiter, as well as the existence of crater chains on the Galilean satellites and our own Moon, strongly suggest that many if not most kilometer-sized objects in the Solar System are rubble piles (Asphaug and Benz 1994, Richardson et al. 1998a). So far, numerical simulations have either been severely limited in the number of particles in the rubble piles (a few hundred), or used questionable approximations to the collisional dynamics. Our method would eliminate both problems. Note that if small objects in the Solar System are indeed rubble piles, there are implications for planetesimal formation as well. For example, tidal breakup would increase the cross section for fragmentation. As work on our simulations progresses, there is no doubt that other interesting areas of application will turn up as well.

\section{ACKNOWLEDGMENTS}

We thank J. Chambers, M. Duncan, J. Hahn, E. Kokubo (who reports on a $N=4000, t=5 \times 10^{5}$ year calculation in this issue), D. Lin, N. Murray, C. Reschke, and F. van den Bosch for valuable discussions and contributions. Comments and suggestions by the reviewers were particularly appreciated. This work was supported in part by the NASA HPCC-ESS and Intel Technology 2000 Programs and a NASA Innovative Research grant. The Model B simulation was carried out partly on the San Diego Supercomputer Center Cray T3E and partly on the NASA Goddard Space Flight Center Cray T3E.

\section{REFERENCES}

Aarseth, S. J., D. N. C. Lin, and P. L. Palmer 1993. Evolution of planetesimals. II. Numerical simulations. Astrophys. J. 403, 351-376.

Anderson, R. J. 1993. Computer science problems in astrophysical simulation. In Silver Jubilee Workshop on Computing and Intelligent Systems, pp. 48-61. Tata McGraw-Hill, New Delhi.

Anderson, R. J. 1996. Tree data structures for N-body simulation. In 37th Symp. Foundations of Comp. Sci., pp. 224-233.

Applegate, J. H., M. R. Douglas, Y. Gursel, G. J. Sussman, and J. Wisdom 1986. The outer Solar System for 200 million years. Astron. J. 92, 176-194.

Asphaug, E., and W. Benz 1994. Density of Comet Shoemaker-Levy 9 deduced by modelling breakup of the parent "rubble pile." Nature 370, 120124. 
Barnes, J., and P. Hut 1986. A hierarchical $O(N \log N)$ force-calculation algorithm. Nature 324, 446-449.

Beaugé, C., and S. J. Aarseth 1990. N-body simulations of planetary formation. Mon. Not. R. Astron. Soc. 245, 30-39.

Bentley, J. L., and J. H. Friedman 1979. Data structures for range searching. Comput. Surv. 11, 397-409.

Boss, A. P. 1997. Giant planet formation by gravitational instability. Science 276, 1836-1839.

Cameron, A. G. W., and W. Benz 1991. The origin of the Moon and the single impact hypothesis. IV. Icarus 92, 204-216.

Chambers, J. E., and G. W. Wetherill 1996. $N$-body simulations of the formation of the inner planets. Bull. Am. Astron. Soc. 28, 1107.

Chambers, J. E., and G. W. Wetherill, 1998. Making the terrestrial planets: $\mathrm{N}$-body integrations of planetary embryos in three dimensions. Icarus $\mathbf{1 3 6}$, 304-327.

Chambers, J. E., G. W. Wetherill, and A. P. Boss 1996. The stability of multiplanet systems. Icarus 119, 261-268.

Dones, L., and S. Tremaine 1993. Why does the Earth spin forward? Science 259, 350-354.

Duncan, M., T. Quinn, and S. Tremaine 1989. The long-term evolution of orbits in the Solar System: A mapping approach. Icarus 82, 402-418.

Goldreich, P., and S. Tremaine 1980. Disk-satellite interactions. Astrophys. J. 241, 425-441.

Gradie, J. C., C. R. Chapman, and E. F. Tedesco 1989. Distribution of taxonomic classes and the compositional structure of the asteroid belt. In Asteroids II (R. P. Binzel, T. Gehrels, and M. S. Matthews, Eds.), pp. 316-335. Univ. of Arizona Press, Tucson.

Greenberg, R., M. Fischer, G. B. Valsecchi, and A. Carusi 1996. Sources of planetary rotation: Mapping planetesimals' contributions to angular momentum. Bull. Am. Astron. Soc. 28, 1108.

Greenberg, R., J. Wacker, C. R. Chapman, and W. K. Hartman 1978. Planetesimals to planets: Numerical simulation of collisional evolution. Icarus 35, $1-26$.

Hayashi, C. 1981. Structure of the solar nebula, growth and decay of magnetic fields and effects of magnetic and turbulent viscosities on the nebula. Prog. Theor. Phys. Suppl. 70, 35-53.

Hut, P., J. Makino, and S. McMillan 1995. Building a better leapfrog. Astrophys. J. 443, L93-L96.

Ida, S., and J. Makino 1992a. $N$-body simulation of gravitational interaction between planetesimals and a protoplanet. I. Velocity distribution of planetesimals. Icarus 96, 107-120.

Ida, S., and J. Makino 1992b. $N$-body simulation of gravitational interaction between planetesimals and a protoplanet. II. Dynamical friction. Icarus $\mathbf{9 8}$, 28-37.

Ida, S., and J. Makino 1993. Scattering of planetesimals by a protoplanet: Slowing down of runaway growth. Icarus 106, 210-227.

Ida, S., R. M. Canup, and G. R. Stewart 1997. Lunar accretion from an impactgenerated disk. Nature 389, 353-357.

Jewell, G. M., and S. G. Alexander 1996. A hybrid statistical/N-body simulation for mid-stage Solar System formation. Bull. Am. Astron. Soc. 28, 1107.

Kokubo, E., and S. Ida 1995. Orbital evolution of protoplanets embedded in a swarm of planetesimals. Icarus 114, 247-257.

Kokubo, E., and S. Ida 1996. On runaway growth of planetesimals. Icarus 123, 180-191.

Kokubo, E., and S. Ida 1998. Oligarchic growth of planetesimals. Icarus 131, 171-178.

Lecar, M., and S. J. Aarseth 1986. A simulation of the formation of the terrestrial planets. Astrophys. J. 305, 564-579.

Levison, H. F., and M. J. Duncan 1994. The long-term dynamical behavior of short-period comets. Icarus 108, 18-36.
Lin, D. N. C., P. Bodenheimer, and D. C. Richardson 1996. Orbital migration of the planetary companion of 51 Pegasi to its present location. Nature 380, 606-607.

Lissauer, J. J. 1987. Timescales for planetary accretion and the structure of the protoplanetary disk. Icarus 69, 249-265.

Lissauer, J. J. 1993. Planet formation. Annu. Rev. Astron. Astrophys. 31, 129174.

Lissauer, J. J., and V. S. Safronov 1991. The random component of planetary rotation. Icarus 93, 288-297.

Lissauer, J. J., and G. R. Stewart 1993. Growth of planets from planetesimals. In Protostars and Planets III (E. H. Levy and J. I. Lunine, Eds.), pp. 1061-1088. Univ. of Arizona Press, Tucson.

Makino, J., and S. J. Aarseth 1992. On a Hermite integrator with Ahmed-Cohen scheme for gravitational many-body problems. Publ. Astron. Soc. Jpn. 44, 141-151.

Malhotra, R. 1993. The origin of Pluto's peculiar orbit. Nature 365, 819-821.

Marcy, G. W., R. P. Butler, S. S. Vogt, D. Fischer, and J. J. Lissauer 1998. A planetary companion to a nearby M4 dwarf, Gliese 876. Astrophys. J. 505, L147-L149.

Murray, N., B. Hansen, M. Holman, and S. Tremaine 1998. Migrating planets. Science 279, 69-72.

Ohtsuki, K., S. Ida, Y. Nakagawa, and K. Nakazawa 1993. Planetary accretion in the solar gravitational field. In Protostars and Planets III (E. H. Levy and J. I. Lunine, Eds.), pp. 1089-1107. Univ. of Arizona Press, Tucson.

Palmer, P. L., D. N. C. Lin, and S. J. Aarseth 1993. Evolution of planetesimals. I. Dynamics: Relaxation in a thin disk. Astrophys. J. 403, 336-350.

Petit, J.-M., A. Morbidelli, and G. Valsecchi 1998. On the excitation of the primordial small body belts. Bull. Am. Astron. Soc. 30, 1453.

Pollack, J. B., O. Hubickyj, P. Bodenheimer, J. J. Lissauer, M. Podolak, and Y. Greenzweig 1996. Formation of the giant planets by concurrent accretion of solids and gas. Icarus 124, 62-85.

Quinn, T. R., N. Katz, J. Stadel, and G. Lake 2000. Time stepping N-body simulations. Astrophys. J., submitted.

Quinn, T. R., S. Tremaine, and M. Duncan 1991. A three million year integration of the Earth's orbit. Astron. J. 101, 2287-2305.

Richardson, D. C. 1993. A new tree code method for simulation of planetesimal dynamics. Mon. Not. R. Astron. Soc. 261, 396-414.

Richardson, D. C. 1994. Tree code simulations of planetary rings. Mon. Not. R. Astron. Soc. 269, 493-511.

Richardson, D. C. 1995. A self-consistent numerical treatment of fractal aggregate dynamics. Icarus 115, 320-335.

Richardson, D. C., W. F. Bottke, Jr., and S. G. Love 1998a. Tidal distortion and disruption of Earth-crossing asteroids. Icarus 134, 47-76.

Richardson, D. C., G. Lake, T. Quinn, J. Stadel 1998b. Direct simulation of planet formation with a million planetesimals: A progress report. Bull. Am. Astron. Soc. 30, 765.

Safronov, V. S. 1969. Evolution of the Protoplanetary Cloud and Formation of the Earth and Planets. Nauka Press, Moscow.

Saha, P., and S. Tremaine 1992. Symplectic integrators for Solar System dynamics. Astron. J. 104, 1633-1640.

Salo, H. 1992. Gravitational wakes in Saturn's rings. Nature 359, 619-621.

Stewart, G. R., and G. W. Wetherill 1988. Evolution of planetesimal velocities. Icarus 74, 542-553.

Strom, S. E., S. Edwards, and M. F. Skrutskie 1993. Evolutionary time scales for circumstellar disks associated with intermediate- and solar-type stars. In Protostars and Planets III (E. H. Levy and J. I. Lunine, Eds.), pp. 837-866. Univ. of Arizona Press, Tucson.

Tanaka, H., and S. Ida 1997. Distribution of planetesimals around a protoplanet in the nebula gas. Icarus 125, 302-316. 
Tremaine, S. 1990. Dark matter in the Solar System. In Baryonic Dark Matter (D. Lynden-Bell and G. Gilmore, Eds.), pp. 37-65. Kluwer Academic, Dordrecht.

Ward, W. R. 1986. Density waves in the solar nebula—Differential Lindblad torque. Icarus 67, 164-180.

Ward, W. R. 1997. Protoplanet migration by nebula tides. Icarus 126, 261-281.

Ward, W. R., and J. M. Hahn 1998. Dynamics of the trans-Neptune region: Apsidal waves in the Kuiper Belt. Astron. J. 116, 489-498.

Weidenschilling, S. J. 1977. The distribution of mass in the planetary system and solar nebula. Astrophys. Space Sci. 51, 153-158.

Wetherill, G. W. 1990. Formation of the Earth. Annu. Rev. Earth Planet. Sci. 18 205-256.
Wetherill, G. W., and G. R. Stewart 1989. Accumulation of a swarm of small planetesimals. Icarus 77, 330-357.

Wetherill, G. W., and G. R. Stewart 1993. Formation of planetary embryos: Effects of fragmentation, low relative velocity, and independent variation of eccentricity and inclination. Icarus 106, 190-204.

Wisdom, J. 1980. The resonance overlap criterion and the onset of stochastic behavior in the restricted three-body problem. Astron. J. 85, 11221133 .

Wisdom, J., and M. Holman 1991. Symplectic maps for the $N$-body problem. Astron, J. 102, 1528-1538.

Yoshida, H. 1990. Construction of higher order symplectic integrators. Phys. Lett. A 150, 262-268. 